\title{
Ka-Band Rotman Lens-Based Retrodirective Beamforming System for Wireless Power Transfer
}

\author{
Hayoung Hong ${ }^{1} \cdot$ Hongsoo Park $^{1} \cdot \mathrm{Kanghyeok} \mathrm{Lee}^{2} \cdot$ Wonwoo Lee ${ }^{2} \cdot \mathrm{Semin}_{\mathrm{Jo}}^{2} \cdot \mathrm{Junhyu}_{\text {Yang }}^{2} \cdot$
}

Changkun Park ${ }^{1,2} \cdot$ Hojin Lee ${ }^{1,2} \cdot$ Sun K. Hong, ${ }^{1,2 *}$

\begin{abstract}
A retrodirective beamforming system (BFS) based on a Rotman lens is proposed for far-field wireless power transfer at Ka-band. The true-time-delay property of the Rotman lens allows for a wideband operation covering 28-38 GHz. The designed BFS comprises a Rotman lens with nine beam ports connected to a nine-element linear Vivaldi array. The proposed BFS is implemented using PCB technology for ease of manufacturing and low-cost processing. The simulated and measured results demonstrate that the proposed BFS can generate nine discrete beams over a scan range of $\pm 45^{\circ}$ with a wide impedance bandwidth.
\end{abstract}

Key Words: Beamforming, Millimeter-Waves, Retrodirective Array, Rotman Lens, Wireless Power Transfer.

\section{INTRODUCTION}

Recent years have seen a surge of interest in millimeter-wave (mm-wave) technologies for applications such as $5 \mathrm{G}$ communications, radars, and the Internet of Things (IoT). Given the characteristics of mm-waves that allow wide bandwidth, small physical system size, and high data rates, it is expected that mmwaves will keep expanding the usability of wireless devices and IoT technologies [1]. This accordingly induces a demand increase for wirelessly charging such devices. In particular, wireless power transfer (WPT) using mm-waves is an attractive solution for wirelessly powering various compact electronic devices. However, for the realization of mm-wave WPT, it would be necessary to overcome inherent drawbacks, such as high path loss and susceptibility to shadowing [2]. These vulnerabilities can be mitigated by utilizing a suitable beamforming system
(BFS) capable of adaptively transmitting wireless power in a direction of interest $[2,3]$. Butler matrix is among the popular types of BFS for mm-wave multibeam applications [4, 5]. However, its narrow bandwidth due to phase shifters makes it hard to utilize for wideband applications. Hence, a BFS with retrodirectivity and wide impedance bandwidth is desired to ensure the selective transmission of waves to a device that requires wireless power. Such a process would involve a beacon signal sent from the device in need, which is then received and processed by the BFS to acquire the necessary information to steer the beam toward the device $[6,7]$.

The Rotman lens is an attractive choice for retrodirective BFS [8], as it features all passive, true-time-delay (TTD) operations. Its geometric structure, which comprises switchable input beam ports and multiple output array ports connected to an antenna array, allows multiple discrete beams to be formed

Manuscript received November 23, 2020 ; Revised March 17, 2021 ; Accepted May 5, 2021. (ID No. 20201123-189J)

${ }^{1}$ School of Electronic Engineering, Soongsil University, Seoul, Korea.

${ }^{2}$ Department of ICT Convergence, Soongsil University, Seoul, Korea.

"Corresponding Author: Sun K. Hong (e-mail: shong215@ssu.ac.kr)

This is an Open-Access article distributed under the terms of the Creative Commons Attribution Non-Commercial License (http://creativecommons.org/licenses/by-nc/4.0) which permits unrestricted non-commercial use, distribution, and reproduction in any medium, provided the original work is properly cited.

(c) Copyright The Korean Institute of Electromagnetic Engineering and Science. 
passively in different angular directions without using active phase shifters. Moreover, TTD caused by the optical paths in the lens facilitates beamforming independent of frequency. Hence, passive beam tracking is possible with retrodirective operation, as the signals received from a target can be retransmitted in the corresponding direction. The Rotman lens has been widely used in many areas, especially in military applications, satellite communication, and wireless networks [9-12]. With its compact structure, lightweight, and cost-effective fabrication, the Rotman lens can be implemented using various materials and fabrication technologies, including microstrips, substrate integrated waveguides (SIWs), ridge gap waveguides (RGWs), and low-temperature co-fired ceramic (LTCC) technology [12$16]$.

\section{DESIGN OF BFS BASED ON ROTMAN LENS}

\section{Operation Methodology of the Rotman Lens}

The Rotman lens is a wide-angle microwave lens that operates as a wideband beamforming network. The design procedure of the Rotman lens starts with defining the operating frequency range, the number of beam ports, and the beam scan range. A typical Rotman lens comprises $M$ beam ports and $N$ array ports with several dummy ports, as depicted in Fig. 1. The circular beam contour on the left side of the Rotman lens has off-axis focal points $F_{1}$ and $F_{2}$ located at angles $\pm \alpha^{0}$ symmetric about the axis, and on-axis focal point $G$ located at $0^{\circ}$ angle. The design equations initiated by Rotman and subsequently developed are based on the optical path length equality between a general ray from one of the foci to the array elements and a center ray from the same focus to the origin [8]. That is,

$$
\overrightarrow{F_{1} P_{l}}+W_{i}+D \sin \alpha=L+l
$$

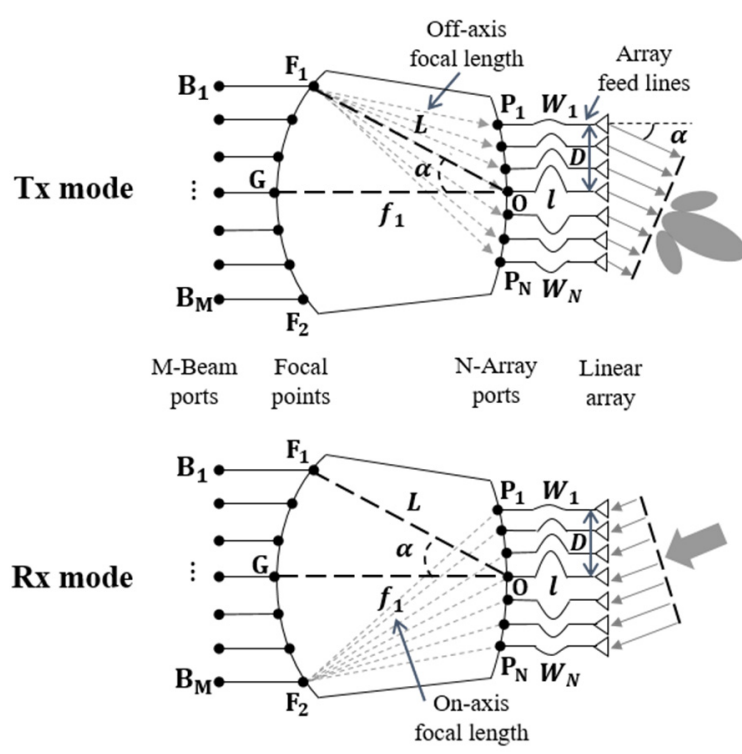

Fig. 1. Configuration and design parameters of Rotman lens.

$$
\begin{gathered}
\overrightarrow{F_{2} P_{l}}+W_{i}-D \sin \alpha=L+l, \\
\overrightarrow{G P_{l}}+W_{i}=f_{1}+l .
\end{gathered}
$$

where $P_{i}$ is the $i^{\text {th }}$ array port, $D$ is the half-width of the antenna array, $\alpha$ is the focal angle, $l$ is the length of the center feed line, and $W_{i}$ is the length of the $i^{\text {th }}$ off-center feed line. $L$ and $f_{1}$ are the off-axis and on-axis focal lengths, respectively. By solving the above equation with the ones given in $[8,17,18]$, the relative lengths of the array feed line and the phase center of the beam and array ports on each contour can be obtained. Here, the TTD is generated by different line lengths of the array feed lines which cause relative phase shifts, inducing a tilted wavefront towards the desired angle during radiation. This TTD property allows for the Rotman lens to be operated over a wide frequency band. The presence of dummy ports can absorb and minimize internal reflections inherently introduced by the lens sidewalls. The curvature design of the sidewalls and the number of dummy ports are carefully chosen to minimize pattern distortion and side lobe level.

Furthermore, reciprocity in the Rotman lens allows retrodirective operation. In a WPT scenario, a beacon signal from the device that requires wireless power enters the Rotman lens through an antenna array, where the waves end up focusing on the beam port corresponding to the angle of incidence. That is, in the receive $(\mathrm{Rx})$ mode, the angle of the incident waves within a scan range of $\pm \alpha^{0}$ determines which beam port can be used in the transmit mode. Therefore, in the transmit $(\mathrm{Tx})$ mode, by exciting the corresponding beam port, wireless power can be selectively transmitted to the device of interest.

\section{Design of the Proposed Rotman Lens}

In the preliminary design stage, the REMCOM Rotman Lens Designer (RLD) is used for parameter studies and frame design. The proposed Rotman lens will operate at a frequency range of $28-38 \mathrm{GHz}$ with a center frequency of $33 \mathrm{GHz}$, over a maximum scan range of $\pm 45^{\circ}$. Since the number of beam ports is associated with angular resolution, nine beam ports are set to fully cover the scan range, as shown in Fig. 2 . These ports can steer the beam discretely at an increment of $11.25^{\circ}$. The lens employs nine array ports with an element spacing of halfwavelength of the center frequency. The lens sidewalls are designed to have eight dummy ports terminated with $50 \Omega$ loads to minimize internal reflections. The proposed Rotman lens is fabricated on a Rogers RO3006C substrate $\left(\varepsilon_{r}=3.38, \tan \delta=\right.$ 0.0027 and thickness $=0.203 \mathrm{~mm}$ ) and the overall size of the lens is $76 \mathrm{~mm} \times 76 \mathrm{~mm}$. Full-wave simulation of the designed Rotman lens is performed using SEMCAD X [19]. The simulated results exhibit good impedance matching $(<-10 \mathrm{~dB})$ at all beam ports, as plotted in Fig. 3. A wide impedance bandwidth of $30.3 \%$ (from $28 \mathrm{GHz}$ to $38 \mathrm{GHz}$ ) is observed, which covers most of the Ka-band. 


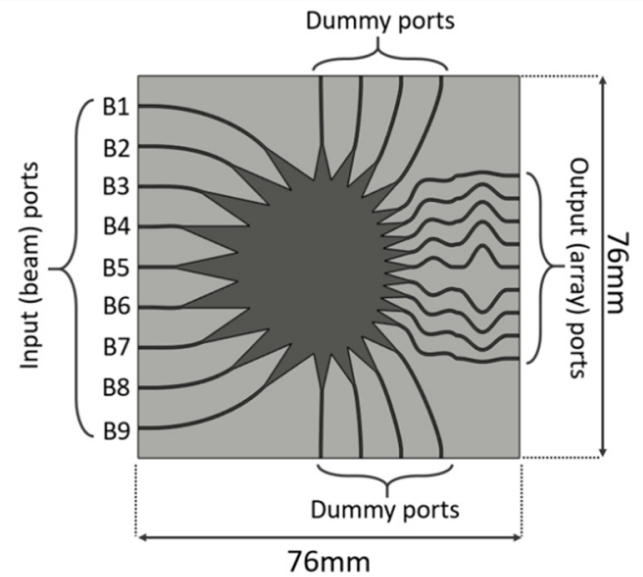

Fig. 2. Geometry of designed Rotman lens.

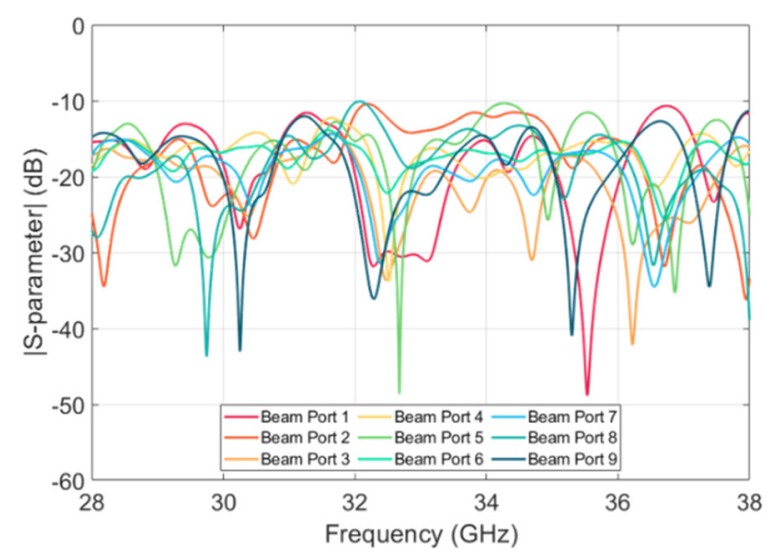

Fig. 3. $S$-parameter of the Rotman lens at beam ports.

\section{Design of the Proposed Rotman Lens}

Due to the wideband characteristics of the Rotman lens, an antenna element with a wide operating bandwidth should be incorporated into the BFS. Here, we are interested in designing a horizontally flat BFS that can radiate vertically polarized waves in the azimuth plane. Note that the antenna elements used with the Rotman lens affect the radial plane and gain of the BFS. For a planar Rotman lens in the horizontal (azimuth) plane, beamforming can be performed vertically or horizontally, depending on the antenna array configuration.

In our work, the Vivaldi antenna was selected due to its inherent wideband and directional characteristics [20, 21]. The end-fire radiation of the Vivaldi antenna is appropriate for beamforming in forward-looking angles. By connecting the Vivaldi elements perpendicular to the Rotman lens, vertically polarized beams can be formed in the azimuth plane, unlike inplane printed Rotman lenses and antennas from previous works $[12,13,16]$.

Fig. 4 shows the geometry of the Vivaldi element designed to operate at the Ka-band. The side edges of the Vivaldi antenna allow edge currents, which increase the sidelobe level and affect the directional control of the main lobe. To mitigate these short-

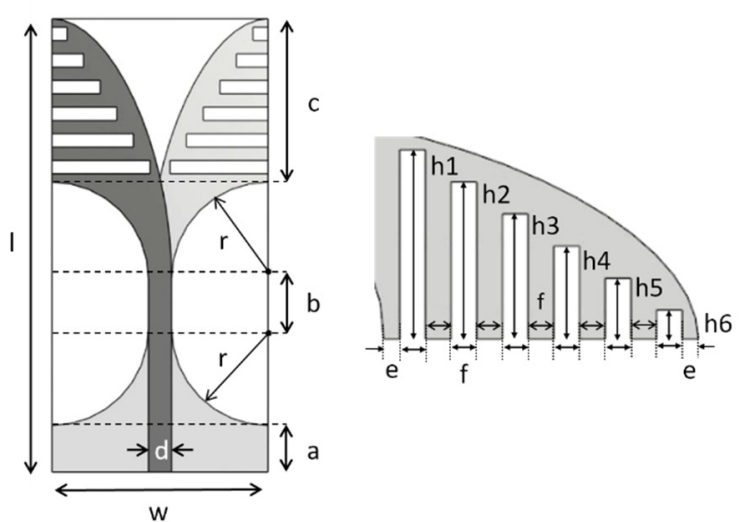

Fig. 4. Geometry of the designed Vivaldi antenna.

comings, regular slot edges (RSEs) are used to mitigate edge currents, thereby alleviating the back and sidelobe levels and increasing the main lobe level by correcting the squint effect [22-24]. Therefore, optimized RSEs are placed on the top and bottom radiators for higher directivity, and detailed dimensional values are presented in Table 1. Six pairs of RSEs are positioned at $0.4 \mathrm{~mm}$ intervals, and the widths of the edges are kept equal, while the lengths decrease gradually. The designed antenna is printed on a Rogers RT/Duroid 5880 laminate $\left(\varepsilon_{r}=2.2, \tan \delta\right.$ $=0.0009$, and thickness $=0.508 \mathrm{~mm}$ ) and the overall size of the Vivaldi is $13.6 \mathrm{~mm} \times 6.5 \mathrm{~mm}$. The bandwidth of the Vivaldi antenna is verified via full-wave simulation and measurement. In Fig. 5(a), the simulated and measured reflection coefficients exhibit good impedance matching over the entire bandwidth (28-38 GHz) of the Rotman lens. Fig. 5(b) shows both simulated and measured gains of a single Vivaldi antenna, which exhibit a similar trend, except that the measured values are generally lower by about $1 \mathrm{~dB}$. This slight decrease in the measured gain can be attributed to the loss associated with the connectors and fabrication. The performance of $\mathrm{mm}$-wave devices can be very sensitive to the soldering and connectors used. By using a better fabrication process and connectors, such losses can be improved.

Table 1. Geometrical parameters of the Vivaldi antenna (unit: $\mathrm{mm}$ )

\begin{tabular}{cccc}
\hline Parameter & Value & Parameter & Value \\
\hline$W$ & 6.5 & $\mathrm{f}$ & 0.4 \\
$L$ & 13.6 & $\mathrm{~h} 1$ & 2.95 \\
$a$ & 1.4 & $\mathrm{~h} 2$ & 2.45 \\
$b$ & 1.5 & $\mathrm{~h} 3$ & 1.95 \\
$c$ & 4.9 & $\mathrm{~h} 4$ & 1.45 \\
$r$ & 2.9 & $\mathrm{~h} 5$ & 0.95 \\
$d$ & 0.7 & $\mathrm{~h} 6$ & 0.45 \\
$e$ & 0.25 & - & - \\
\hline
\end{tabular}




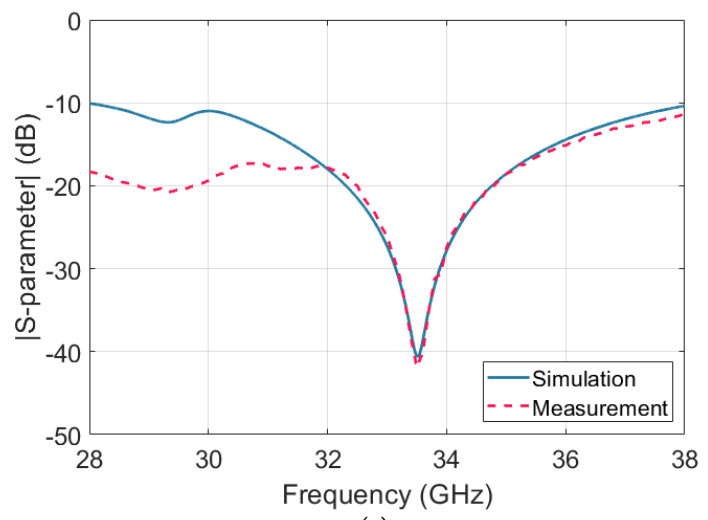

(a)

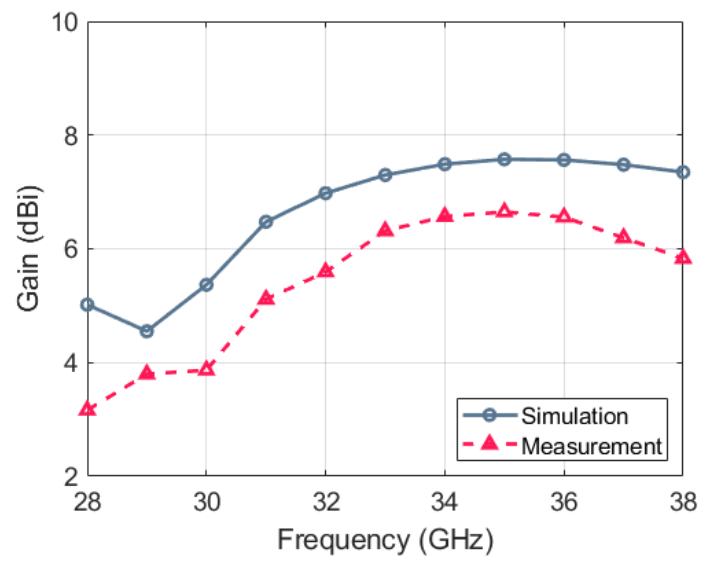

(b)

Fig. 5. The simulated and measured performance of designed Vivaldi antenna: (a) return loss and (b) antenna gain.

\section{SIMULATED AND MEASURED RESULTS OF BFS}

The simulated model of the proposed BFS is shown in Fig. 6(a). The Rotman lens in connection with a nine-element Vivaldi array is simulated to demonstrate the beam steering capability at nine different angles. The simulated beams at 33 $\mathrm{GHz}$ are plotted in Fig. 6(b). With an azimuth scan range of $\pm 45^{\circ}$, the BFS produces satisfactory radiation patterns at all beam ports. The patterns generated by exciting the beam ports one at a time are shown in Fig. 6, and the resulting beams point to $-46^{\circ},-32^{\circ},-22^{\circ},-11^{\circ}, 0^{\circ}, 11^{\circ}, 21^{\circ}, 32^{\circ}$, and $45^{\circ}$, respectively. There is a slight beam-pointing error for each beam of up to $1.75^{\circ}$, some of which may be attributed to quantization when extracting the patterns from simulation. Further, the beam pointing error is within the $3 \mathrm{~dB}$ beamwidth of each beam, indicating that all nine beams still properly cover the designed scan range.

To test the proposed BFS via measurement, the Rotman lens is combined with the Vivaldi array, as shown in Fig. 7(a). In Fig. 7(b), the measured reflection coefficients of the BFS are plotted, which demonstrate a good impedance matching $(<-10 \mathrm{~dB})$ over the bandwidth of interest at all input beam ports, validating the simulated results.

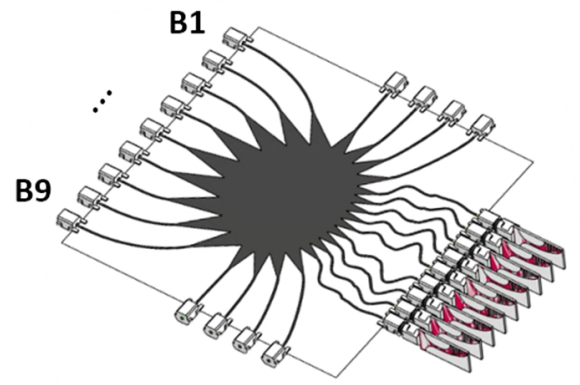

(a)

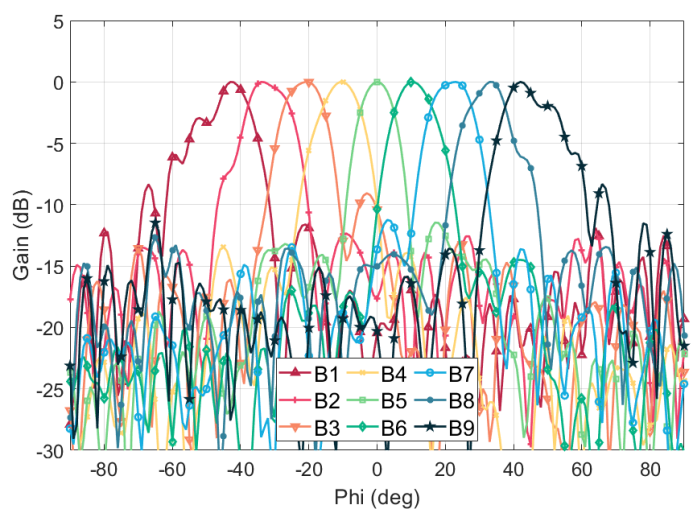

(b)

Fig. 6. BFS based on designed Rotman lens. (a) Lay-out of the proposed BFS with Vivaldi antennas. (b) Simulated radiation patterns at $33 \mathrm{GHz}$.

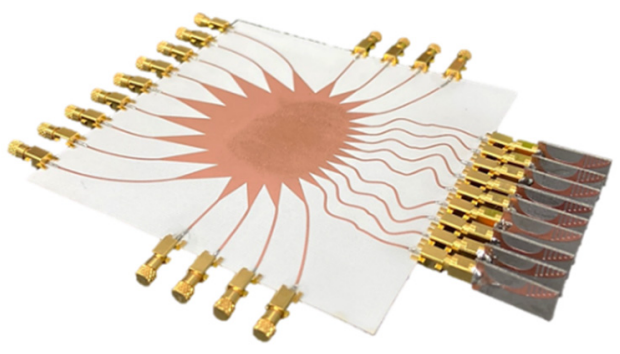

(a)

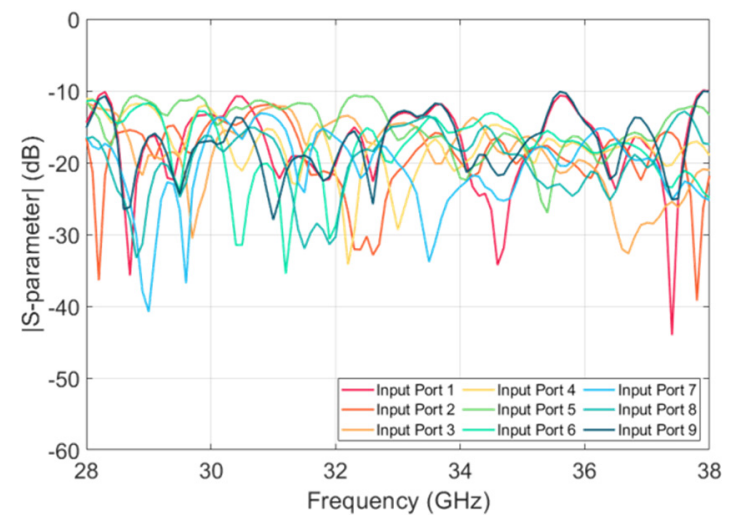

(b)

Fig. 7. The fabricated Rotman lens and its impedance characteristics.

(a) The fabricated Rotman lens with a Vivaldi array. (b) Measured reflection coefficients at beam ports. 
The radiation characteristics of the fabricated BFS are measured in an anechoic chamber. The BFS is mounted on a plastic jig for measurement in the chamber, as shown in Fig. 8. The radiation pattern in the azimuth is measured by rotating the positioner at a $1^{\circ}$ increment.

To measure the radiation patterns of the proposed BFS from excitation at each beam port, all the non-excited ports are terminated with $50 \Omega$ loads. In Fig. 9(a)-(c), the measured radiation

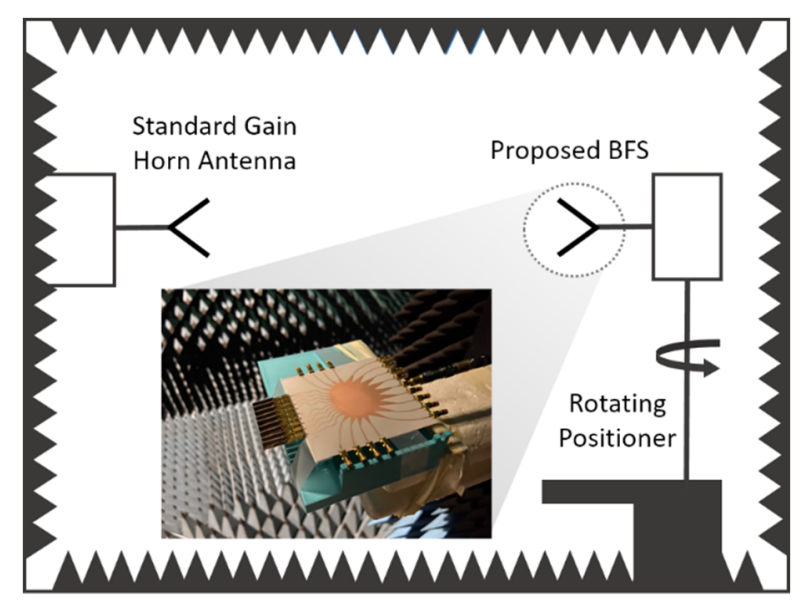

Fig. 8. Illustration of measurement setup in anechoic chamber.

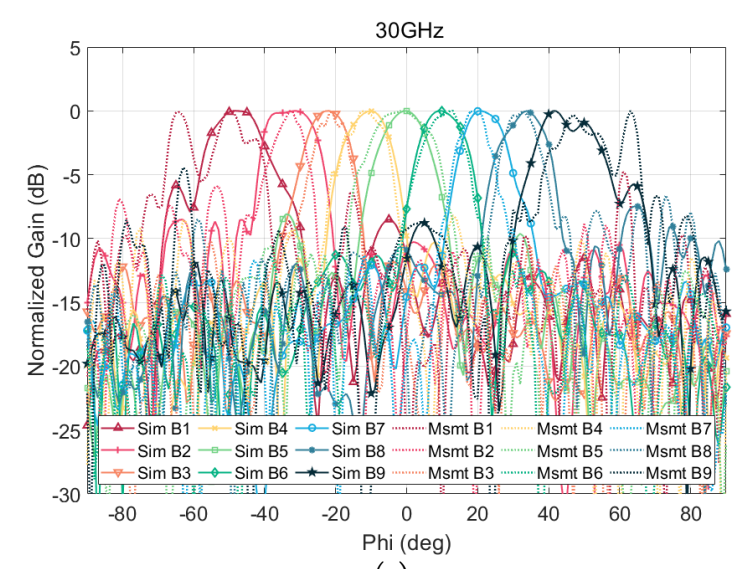

(a)

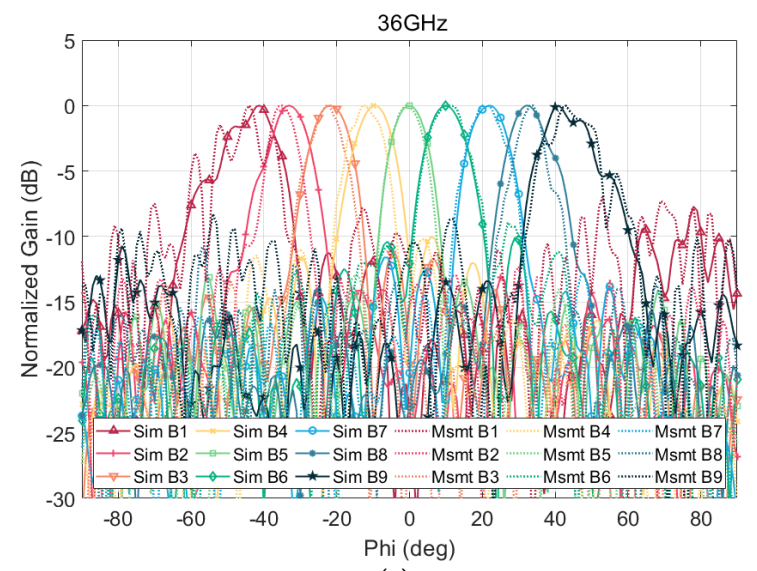

(c) patterns are plotted in comparison with the simulated patterns at $30 \mathrm{GHz}, 33 \mathrm{GHz}$, and $36 \mathrm{GHz}$, respectively. It is demonstrated that the simulated and measured results are generally in good agreement. The measured results show that beams are generated at peak angles of $-43^{\circ},-34^{\circ}-20^{\circ},-16^{\circ}, 0^{\circ}, 12^{\circ}, 20^{\circ}, 34^{\circ}$, and $43^{\circ}$ at $33 \mathrm{GHz}$. Compared with the simulated results, the maximum difference of the peak angles is $5^{\circ}$, which is within the 3 $\mathrm{dB}$ beamwidth. Moreover, there are relatively high sidelobes in some of the measured patterns for the farthest tilt angles in particular. These discrepancies in radiation patterns may be caused by unwanted wave reflections and interferences from the jig or terminator/connector and other objects in the apparatus.

For the maximum realized gain, the simulated and measured results also follow a similar trend within the frequency ranges, as plotted in Fig. 9(d). The maximum gain of the center beam (B5 excited) is $12.5 \mathrm{~dB}$ at $33 \mathrm{GHz}$ from the simulation and $11.3 \mathrm{~dB}$ at $36 \mathrm{GHz}$ from the measurement. However, the measured gain values are generally lower by a few $\mathrm{dB}$ across the bandwidth. Such a discrepancy between the measured and simulated gain can be explained by unaccounted dielectric loss, slight misalignment of the Vivaldi array, fabrication sensitivity in mm-wave frequencies, and performance variations in each Vivaldi element. Our analysis indicates that the dielectric loss of the substrate

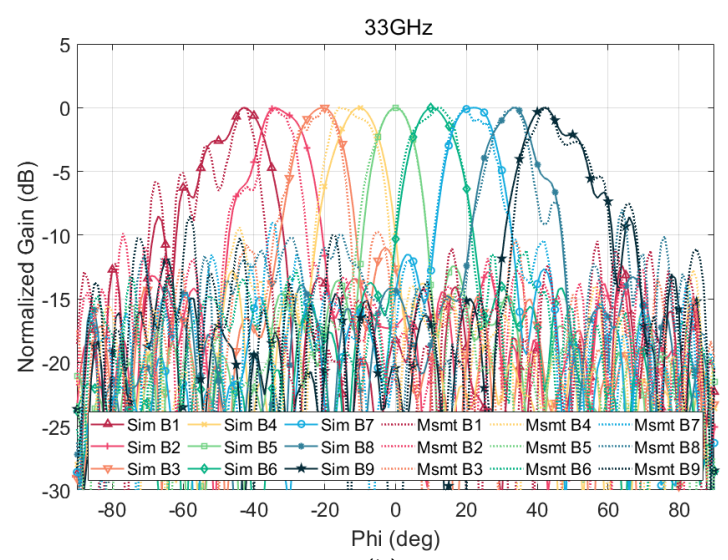

(b)

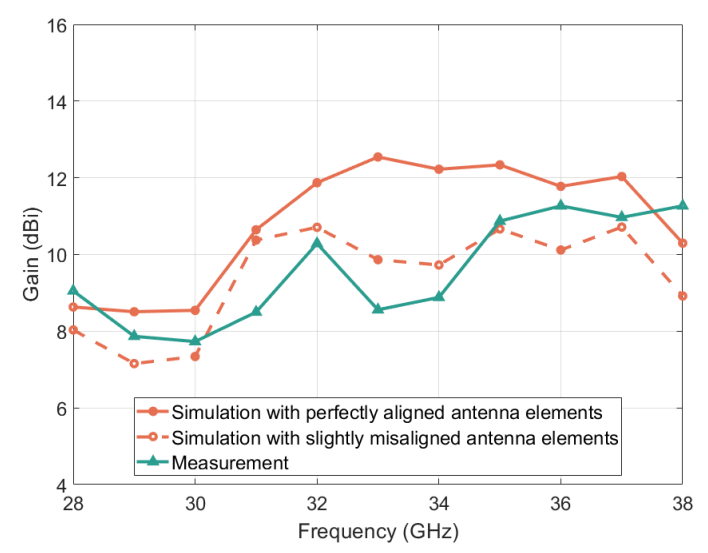

(d)

Fig. 9. Simulated and measured realized gain at (a) $30 \mathrm{GHz}$, (b) $33 \mathrm{GHz}$ and (c) $36 \mathrm{GHz}$, and (d) maximum realized gain values at $\mathrm{B} 5$. 
and blind connection with SMPM connectors/adapters seem to contribute to a gain loss of about $2-3 \mathrm{~dB}$. Since the alignment of the array antenna significantly influences the BFS's radiated direction, minor misalignment can also cause phase incoherency in the desired direction, prompting a loss of gain. These factors of discrepancy are demonstrated by simulating the proposed structure with slightly misaligned array elements and connectors. In Fig. 9(d), the dashed orange trace is the simulated gain results with misaligned antenna elements and connectors, which shows a similar trend to the measured gain results, indicating that antenna misalignment has a decisive effect on loss of gain. In addition, deviations in the measured gain performance of each of the nine antennas are responsible for the additional gain losses. Nevertheless, the overall results suggest that the proposed BFS properly exhibits beamforming capability as desired in the scan range and frequency band of interest.

Through further optimization in design/configuration, gain losses can potentially be minimized using robust connectors, such as $2.4 \mathrm{~mm}$ or $\mathrm{K}$ connectors, rather than blind mate connectors (SMPM) used here. Moreover, further gain improvement can be achieved by improving the gain of the array elements.

\section{CONCLUSION}

A BFS based on the Rotman lens with a Vivaldi array is presented for far-field wireless power transfer at a Ka-band. The proposed system comprises a Rotman lens with nine-beam ports and a nine-element Vivaldi array. Both the Rotman lens and Vivaldi antennas are tested via numerical simulation and measurement. The overall results indicate that the proposed BFS exhibits wideband impedance and radiation characteristics over a bandwidth of $28-38 \mathrm{GHz}$ and beam-steering capability over a scan range of $\pm 45^{\circ}$. With the retrodirective property of the Rotman lens, the proposed BFS has good potential as a suitable means for far-field WPT applications in the $\mathrm{mm}$-wave band. Since the proposed BFS is configured to have antenna elements easily attached and detached, it is also possible to reconfigure the BFS with more directive antenna elements for additional gain.

This work was supported by the Office of Naval Research Global (Grant No. N62909-19-1-2049).

\section{REFERENCES}

[1] T. S. Rappaport, S. Sun, R. Mayzus, H. Zhao, Y. Azar, K. Wang, et al., "Millimeter wave mobile communications for
5G cellular: It will work!," IEEE Access, vol. 1, pp. 335-349, 2013.

[2] S. Rangan, T. S. Rappaport, and E. Erkip, "Millimeterwave cellular wireless networks: potentials and challenges," Proceedings of the IEEE, vol. 102, no. 3, pp. 366-385, 2014.

[3] Y. Zeng, B. Clerckx, and R. Zhang, "Communications and signals design for wireless power transmission," IEEE Transactions on Communications, vol. 65, no. 5, pp. 22642290, 2017.

[4] Y. Cao, K. S. Chin, W. Che, W. Yang, and E. S. Li, "A compact $38 \mathrm{GHz}$ multibeam antenna array with multifolded butler matrix for 5G applications," IEEE Antennas and Wireless Propagation Letters, vol. 16, pp. 2996-2999, 2017.

[5] J. W. Lian, Y. L. Ban, C. Xiao, and Z. F. Yu, "Compact substrate-integrated $4 \times 8$ Butler matrix with sidelobe suppression for millimeter-wave multibeam application," IEEE Antennas and Wireless Propagation Letters, vol. 17, no. 5, pp. 928-932, 2018.

[6] Y. Li and V. Jandhyala, "Design of retrodirective antenna arrays for short-range wireless power transmission," IEEE Transactions on Antennas and Propagation, vol. 60, no. 1, pp. 206-211, 2012.

[7] A. Massa, G. Oliveri, F. Viani, and P. Rocca, "Array designs for long-distance wireless power transmission: state-ofthe-art and innovative solutions," Proceedings of the IEEE, vol. 101, no. 6, pp. 1464-1481, 2013.

[8] W. Rotman and R. Turner, "Wide-angle microwave lens for line source applications," IEEE Transactions on Antennas and Propagation, vol. 11, no. 6, pp. 623-632, 1963.

[9] O. Kilic and S. J. Weiss, "Rotman lens applications for the US Army: a review of history, present, and future," URSI Radio Science Bulletin, vol. 2010, no. 332, pp. 10-23, 2010.

[10] Y. J. Cheng, W. Hong, K. Wu, Z. Q. Kuai, C. Yu, J. X. Chen, J. Y. Zhou, and H. J. Tang, "Substrate integrated waveguide (SIW) Rotman lens and its Ka-band multibeam array antenna applications," IEEE Transactions on Antennas and Propagation, vol. 56, no. 8, pp. 2504-2513, 2008.

[11] R. Rotman, M. Tur, and L. Yaron, "True time delay in phased arrays," Proceedings of the IEEE, vol. 104, no. 3, pp. 504-518, 2016.

[12] M. A. Hassanien, R. Hahnel, and D. Plettemeier, "A novel electronically wideband steering system using Rotman lens for 5G applications at $28 \mathrm{GHz}$," in Proceedings of the 12th European Conference on Antennas and Propagation (EuCAP), London, UK, 2018, pp. 1-5.

[13] A. Eid, J. G. Hester, and M. M. Tentzeris, "Rotman lensbased wide angular coverage and high-gain semipassive architecture for ultralong range mm-wave RFIDs" IEEE 
Antennas and Wireless Propagation Letters, vol. 19, no. 11, pp. 1943-1947, 2020.

[14] K. Tekkouk, M. Ettorre, L. Le Coq, and R. Sauleau, "Multibeam SIW slotted waveguide antenna system fed by a compact dual-layer Rotman lens," IEEE Transactions on Antennas and Propagation, vol. 64, no. 2, pp. 504-514, 2016.

[15] F. C. Suarez, D. N. Mendez, and M. Baquero-Escudero, "Rotman lens with ridge gap waveguide technology for millimeter wave applications," in Proceedings of 2013 7th European Conference on Antennas and Propagation (EuCAP), Gothenburg, Sweden, 2013, pp. 4006-4009.

[16] A. Attaran, R. Rashidzadeh, and A. Kouki, "60 GHz low phase error Rotman lens combined with wideband microstrip antenna array using LTCC technology," IEEE Transactions on Antennas and Propagation, vol. 64, no. 12, pp. 5172-5180, 2016.

[17] R. C. Hansen, "Design trades for Rotman lenses," IEEE Transactions on Antennas and Propagation, vol. 39, no. 4, pp. 464-472, 1991.

[18] P. Simon, "Analysis and synthesis of Rotman lenses," in Proceedings of the 22nd ALAA International Communications Satellite Systems Conference \& Exhibit 2004 (ICSSC), Monterey, CA, 2004.

[19] Schmid \& Partner Engineering, "SEMCAD X Matter

\section{Hayoung Hong}

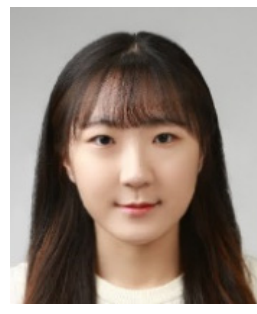

received her B.S. degrees in Electronic Engineering from Soongsil University, Seoul, South Korea, in 2020, and is currently pursuing her M.S. degree in Electronic Engineering at Soongsil University. Her research interests include wireless power transfer, antennas, radars, microwaves, and millimeter-wave devices.

\section{Hongsoo Park}

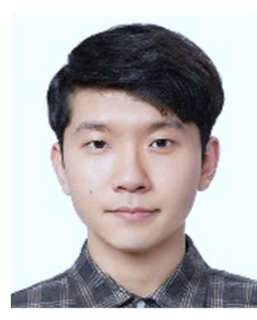

received his B.S. (with honors) and M.S. degrees in Electronic Engineering from Soongsil University, Seoul, South Korea, in 2019 and 2021, respectively. He is currently pursuing his Ph.D. degree at Soongsil University. His research interests include wireless power transfer, antennas, microwave and millimeterwave devices, electromagnetic time-reversal, and electromagnetics in complex environments. horn full-wave 3D electromagnetic (EM) simulation software," 2020 [Online]. Available: https://speag.swiss/ products/semcad/overview/.

[20] J. D. S. Langley, P. S. Hall, and P. Newham, "Balanced antipodal Vivaldi antenna for wide bandwidth phased arrays," IEE Proceedings-Microwaves, Antennas and Propagation, vol. 143, no. 2, pp. 97-102, 1996.

[21] M. Moosazadeh, S. Kharkovsky, and J. T. Case, "Microwave and millimetre wave antipodal Vivaldi antenna with trapezoid-shaped dielectric lens for imaging of construction materials," IET Microwaves, Antennas \& Propagation, vol. 10, no. 3, pp. 301-309, 2016.

[22] G. Teni, N. Zhang, J. Qiu, and P. Zhang, "Research on a novel miniaturized antipodal Vivaldi antenna with improved radiation," IEEE Antennas and Wireless Propagation Letters, vol. 12, pp. 417-420, 2013.

[23] A. M. De Oliveira, M. B. Perotoni, S. T. Kofuji, and J. F. Justo, "A palm tree antipodal Vivaldi antenna with exponential slot edge for improved radiation pattern," IEEE Antennas and Wireless Propagation Letters, vol. 14, pp. 1334-1337, 2015.

[24] H. Liu, W. Yang, A. Zhang, S. Zhu, Z. Wang, and T. Huang, "A miniaturized gain-enhanced antipodal Vivaldi antenna and its array for $5 \mathrm{G}$ communication applications," IEEE Access, vol. 6, pp. 76282-76288, 2018.

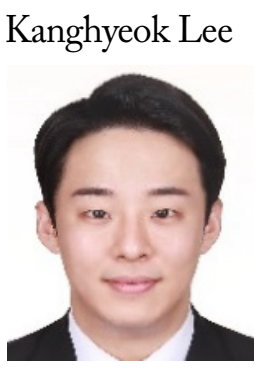

received his B.S. and M.S. degrees in Electronic Engineering from Soongsil University, Seoul, South Korea, in 2019 and 2021, respectively. He is currently with Agency for Defense Development (ADD). His research interests include wireless power transfer, metamaterials, microwave and millimeter devices, antennas, and radar.

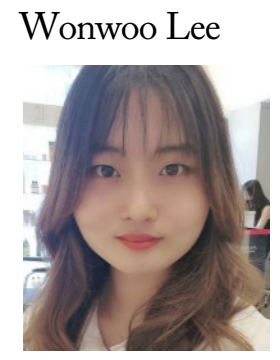

received her B.S. and M.S. degrees in Electronic Engineering from Soongsil University, Seoul, South Korea, in 2016 and 2018, respectively. She is currently pursuing her Ph.D. degree at Soongsil University. Her research interests include microwave energy-harvesting metamaterial-based chemical sensors, $\mathrm{mm}$-wave electromagnetic focusing on meta-surface lens, and terahertz meta-material design, fabrication, and analysis. 


\section{Semin Jo}

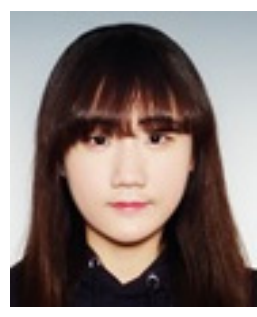

received her B.S. degree in Electronic Engineering from Soongsil University, Seoul, South Korea, in 2019 , and is currently pursuing graduate studies in Information Communication Convergence Technology under the M.S. program at Soongsil University. Her research interests include wireless power transfer, microwave meta-material design, fabrication, and analysis.

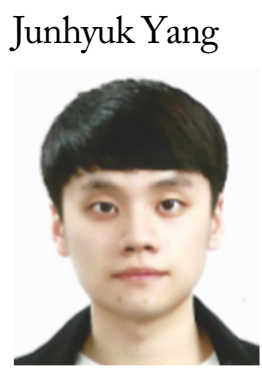

received his B.S. and M.S degrees in Electronic Engineering from Soongsil University, Seoul, South Korea, in 2019 and 2021, respectively. He is currently with Samsung Electronics. His research interests include wireless power transfer and millimeter-wave integrated circuits.

\section{Changkun Park}

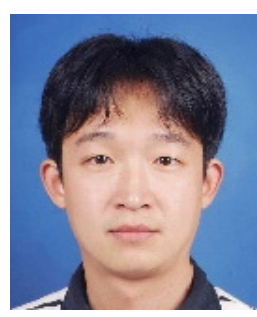

received his B.S., M.S., and Ph.D. degrees in Electrical Engineering from the Korea Advanced Institute of Science and Technology (KAIST), Daejeon, Korea, in 2001, 2003, and 2007, respectively. In September 2009, he joined the Faculty of the School of Electronic Engineering, Soongsil University, Seoul, Korea. His research interests include RF and $\mathrm{mm}-$ wave circuits, RF CMOS power amplifiers, and wireless chip-to-chip communication and power transfers.

\section{Hojin Lee}

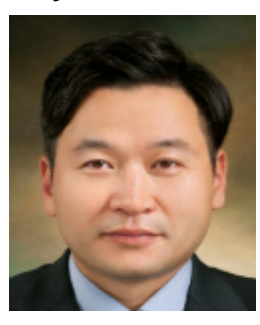

received his B.S. and M.S. degrees in Electrical Engineering from Hanyang University, Seoul, South Korea, in 1996 and 1998, respectively, and a Ph.D. degree in Electrical Engineering and Computer Science from the University of Michigan, Ann Arbor, MI, USA, in 2008. He is currently an Associate Professor in the School of Electronic Engineering at Soongsil University, Seoul, South Korea. His current research interests include display circuit design, OLED fabrication, chemical sensor development, ionic polymer-based actuator, $\mathrm{mm}$-wave and terahertz meta-material, and wireless power transfer for bioimplantable devices. He has authored/coauthored more than 40 research papers in refereed journals and conference papers presented at international conferences.

\section{Sun K. Hong}

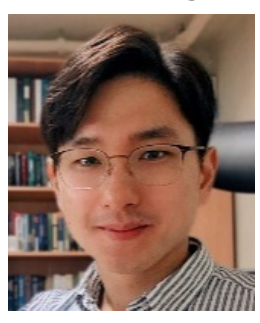

received his B.S. degree in Electrical Engineering from the University of Maryland, College Park, MD, USA, in 2005, and his M.S. and Ph.D. degrees in Electrical Engineering from Virginia Tech, Blacksburg, VA, USA, in 2008 and 2012, respectively. From 2005 to 2015, he was a Research Engineer with the U.S. Naval Research Laboratory. From 2015 to 2017, he was an Assistant Professor in the Department of Electrical and Computer Engineering, Rose-Hulman Institute of Technology, Terre Haute, IN, USA. In 2017, he joined the School of Electronic Engineering at Soongsil University in Seoul, where he is an Assistant Professor. His current research interests include wireless power transfer, electromagnetic waves in complex propagation environments, detection of nonlinear devices, radars, and high-power electromagnetics. 